\title{
Interactive Transmission Line Computer Program for Undergraduate Teaching
}

\author{
Christopher W. Trueman, Senior Member, IEEE
}

\begin{abstract}
Distributed-circuit analysis using transmission lines is a standard topic in undergraduate education in electrical engineering and computer engineering. This paper presents a simple menu-driven program that students can use to explore the behavior of transmission line circuits, and as a computational laboratory for verifying their solutions to homework problems. At the introductory level the program demonstrates traveling waves and standing waves. Smith-chart calculations of impedance and reflection coefficient can be associated with the standing-wave pattern on the transmission line. A branching circuit can be solved, including impedance matching with a quarter-wave transformer. At the intermediate level, students can use the program to test their designs for two- and three-step Chebyshev transformers, and for impedance matching using single, double and triple-stub circuits. The program makes easy the calculation of the bandwidth of a design by displaying the return loss or transmission loss as a function of frequency. Low-pass and bandstop filters can be solved. The program encourages students to explore the behavior of the voltages and currents on the lines as the frequency is changed to gain insight into the operation of transformers, matching schemes and filters.
\end{abstract}

Index Terms-Filters, Smith chart, stub matching, teaching software, transmission lines, travelling waves.

\section{INTRODUCTION}

$\mathbf{S}$ TUDENTS entering a "fields and waves" course based on textbooks such as Kraus [1] or Paul and Nasar [2] are already fluent in the analysis of lumped circuits in the sinusoidal steady-state, using phasors and impedance. The "fields and waves" course replaces the simple wires interconnecting the components with cables having distributed inductance and capacitance. The wires become "transmission lines" carrying traveling waves. The simple voltages and currents of lumped circuit analysis become functions of position. New concepts are introduced in rapid succession: traveling wave, incident wave, reflected wave, reflection coefficient, standing wave, matched load, and standing-wave ratio. The Smith chart provides insight into the behavior of the reflection coefficient and input impedance with changing cable length. The homework exercises usually consist of computing input impedance and reflection coefficient, standing-wave ratio, and power delivered to the load. As students progress, techniques are introduced for creating matched loads, such as the quarter-wave transformer

Manuscript received March 2, 1998; revised October 20, 1999.

The author is with the Department of Electrical and Computer Engineering, Concordia University, Montreal, Quebec Canada H4B 1R6 (e-mail: trueman@ece.concordia.ca).

Publisher Item Identifier S 0018-9359(00)01755-6. and stub matching. The Smith chart provides a graphical solution to the complex problem of choosing stub lengths to achieve matched conditions.

This paper presents a simple computer program called TRLINE (TRansmission LINE) ${ }^{1}$ which supports the teaching of both elementary transmission line concepts and intermediate topics such as stub matching and transformer design. The TRLINE program provides a computational laboratory to allow students to verify their answers to homework exercises and explore in greater depth the transmission-line phenomena dealt with in class and in the homework. TRLINE permits a quick evaluation of the bandwidth of transformers, stub-matching circuits and filters, and so permits the students to develop an awareness of frequency dependence early in their studies. This paper describes each of TRLINE's functions and shows how these can be used as an aid in teaching about transmission line circuits.

Because TRLINE is easy to use, the reader will learn how to operate the program as its functions are described in this paper. The program presents the user with a series of menus, with menu items or "buttons" written in red. Clicking the mouse on any red text string in the program invokes that function. Starting the program gets the "circuit template menu" of Fig. 1, inviting the student to choose a problem for investigation. (The black-andwhite diagrams in this paper do not distinguish the red menu choices from the other text.) Rather than try to teach the student to construct circuits interactively, TRLINE simply presents a choice of built-in circuits, allowing students to get results with the program almost instantly. For convenience in using the program in the classroom, the transformers, stub matching circuits and filters come preset with the frequency, line lengths, and impedances chosen to demonstrate the operation of the circuit.

Choosing a circuit in the template menu, such as "transmission line with generator and load," produces the main menu of Fig. 2. A circuit schematic appears across the center, with "properties buttons" at the top for changing the values of the circuit parameters. Many of the menus in TRLINE include the "properties buttons" so that line lengths and impedances and load values can be changed readily. For example, clicking the mouse on "Line \#1" permits the user to set the length, characteristic resistance, and propagation speed on the transmission line. The "action buttons" for analyzing the circuit are listed at the screen bottom and the corresponding functions are described below. Like most menus in TRLINE, there is an "exit" button at the lower right-hand corner of the screen. In the main menu this button quits the program, but in the submenus it returns to the

\footnotetext{
${ }^{1}$ TRLINE is available from the author for no cost at http://www.ece.concordia.ca/ trueman/trline/index.htm
} 


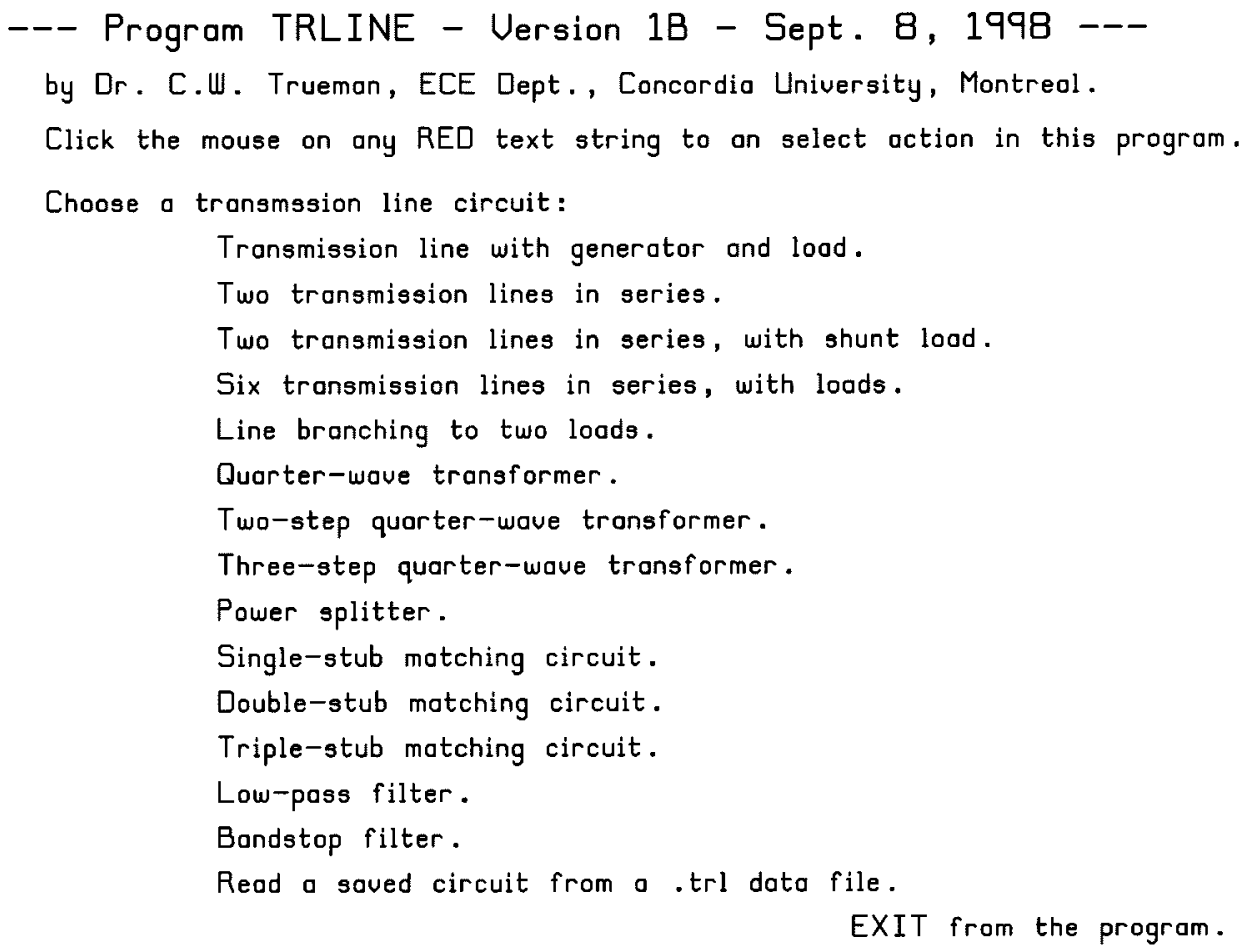

Fig. 1. Program TRLINE offers a variety of built-in circuits on the "circuit template menu."
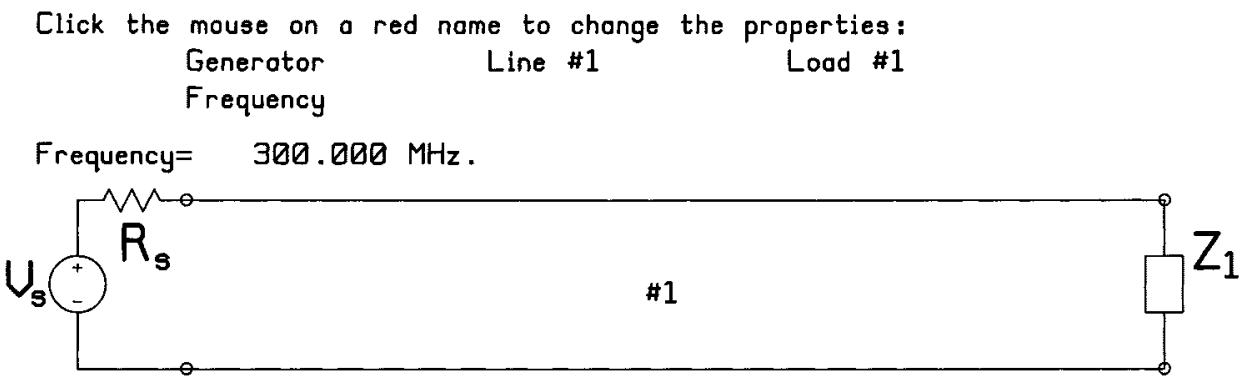

Choose on oction by clicking the mouse on a red text string:

Plot $U(z)$ or $I(z)$, and find the USURR.

Drow o Smith Chart.

Find voltages, currents, and power.

Plot a parameter as a function of the frequency.

Save the circuit to a dato file.

Choose a new circuit.

EXIT from the program.

Fig. 2. The "main menu" gives the user access to the principal functions of the program.

main menu. The following section explains the program's functions and how they can be used to support classroom teaching and homework exercises.

\section{TEACHING THE BASICS}

\section{A. Traveling Waves, Standing Waves and Phase}

The simple circuit of Fig. 2 can be used to demonstrate the basic behavior of waves on transmission lines. Click the mouse on "Plot $V(z) \cdots$ " to get the "transmission line menu" of Fig. 3. By graphing the voltage amplitude and phase as a function of distance along the transmission line, the program helps students to learn to associate phase behavior with the standing-wave pattern. Thus clicking on "Plot the voltage including phase" in Fig. 3 draws the voltage amplitude with a solid line and the phase with a dashed line as in Fig. 4. In part (a) the load is matched so the phase of the voltage is progressive with distance, becoming more and more negative as the wave travels along the line. The wave is a "pure" traveling wave from left to right. The graph helps students to associate "constant-amplitude progressive-phase" strongly with "traveling wave." In part (b) the load is a short circuit, and the amplitude shows a "standing 


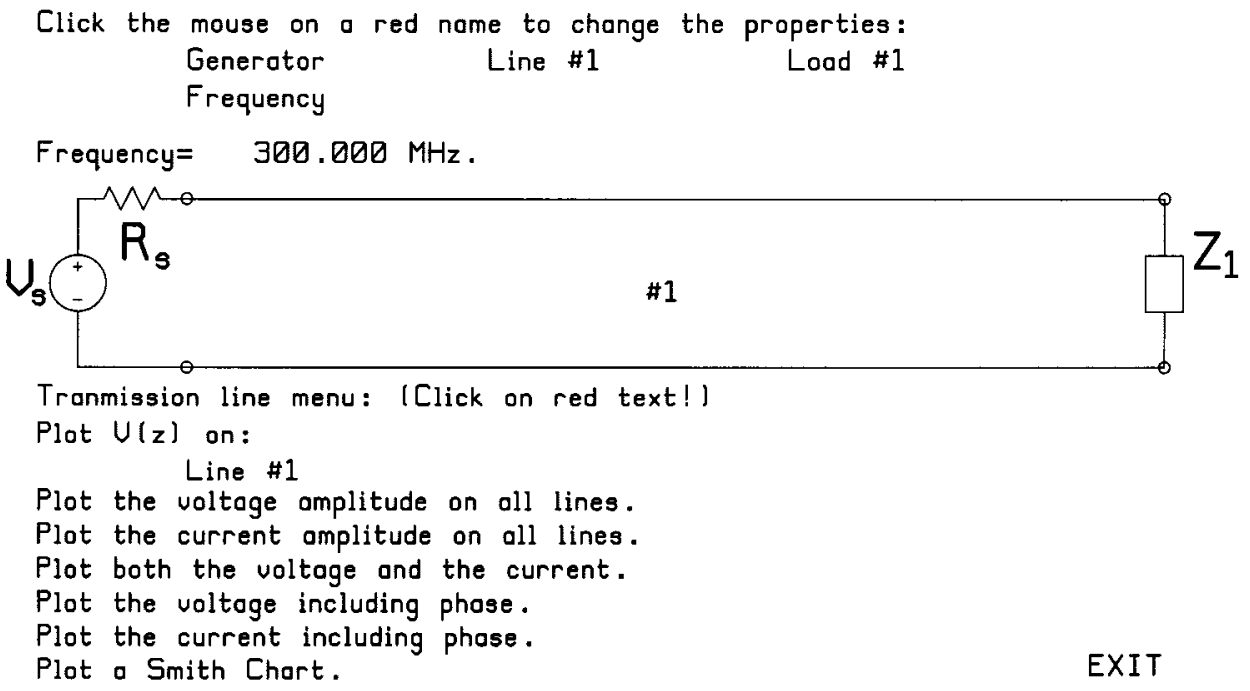

Fig. 3. The "transmission line" is used to plot the voltage or current as a function of position on the transmission lines.

wave" with sharp nulls. Now the phase is constant with distance, except for abrupt reversals at the nulls. Students should learn to associate "constant phase with sharp reversals" with a standing wave. Part (c) uses a 10- $\Omega$ load. The voltage amplitude is a standing-wave pattern but the minima are not "nulls." The phase shows a downward trend with distance, with gradual reversals across the minima in the standing-wave pattern. Comparing parts (a)-(c) supports the idea that in general the wave pattern on a transmission line is the sum of a pure standing wave plus a pure traveling wave.

\section{B. Voltage and Current Standing Wave Patterns}

Click "Plot both the voltage and the current" in Fig. 3 to compare the voltage standing wave to the current standing wave as in Fig. 5, for a line of characteristic impedance $50 \Omega$ and a load of $200 \Omega$. Where the current standing-wave pattern has maxima, the voltage pattern has minima and vice-versa. Because the load impedance is real and greater than the line impedance the load is a voltage maximum. The program reports the standing-wave ratio, equal to four, in the upper left corner of the screen. Students can use the program to explore the changes in the standing-wave pattern as the load impedance is varied from a short circuit, through a match, to an open-circuit. Also, the changes in the positions of the maxima and minima can be explored as the load impedance is changed from resistive to highly reactive.

\section{Positions of the Voltage Maxima and Minima}

A customary homework exercise asks the student to determine the distance from the load to the first standing-wave minimum or maximum, usually for a complex-valued load. The transmission line menu of Fig. 3 supports this exercise by offering a labeled graph of the magnitude of $V(z)$ as a function of distance along the line, including "markers" to read back values. Clicking the mouse on the (red) "Line \#1" button below "Plot $V(z)$ " gets the voltage standing-wave graph of Fig. 6. To move a marker, click the mouse on "Marker \#1"(again in red) and then click again on the new position for the marker. The program re-

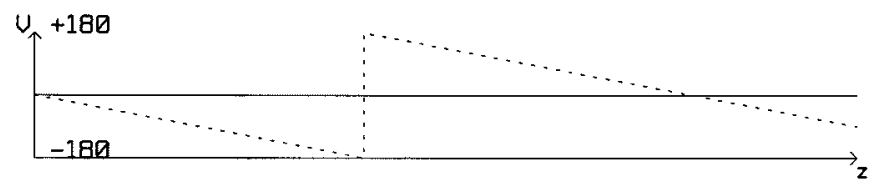

(a)

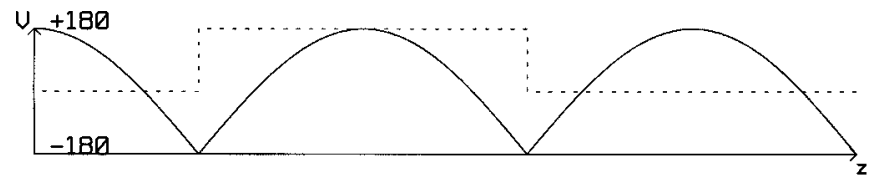

(b)

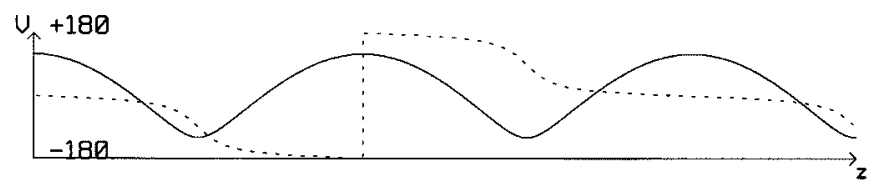

(c)

Fig. 4. The amplitude of the voltage (solid) and of the current (dashed) as a function of distance along the transmission line.

ports the position of the marker from the beginning of the line and the value of the voltage amplitude, in the upper right-hand corner of the screen. The distance between the markers is also reported, so that the student can verify that the minima are half a wavelength apart, as are the maxima. By positioning marker $\# 2$ at the load, and marker \#1 at the nearest voltage minimum, the distance of the minimum from the load can be read. Students can enter their line and load impedances into the program, and then verify that their positions for the maxima and minima agree with those found by hand calculation.

\section{THE SMITH CHART}

Students are usually asked to compute the input impedance of the simple circuit of Fig. 3 both by evaluating the standard formula, and by using the Smith chart [1], [2]. Clicking the mouse on "Smith chart" in either Figs. 2 or 3 gets the "Smith chart" menu of Fig. 7. The "properties buttons" at the 
Line \# 1 USWR $=4.0000$

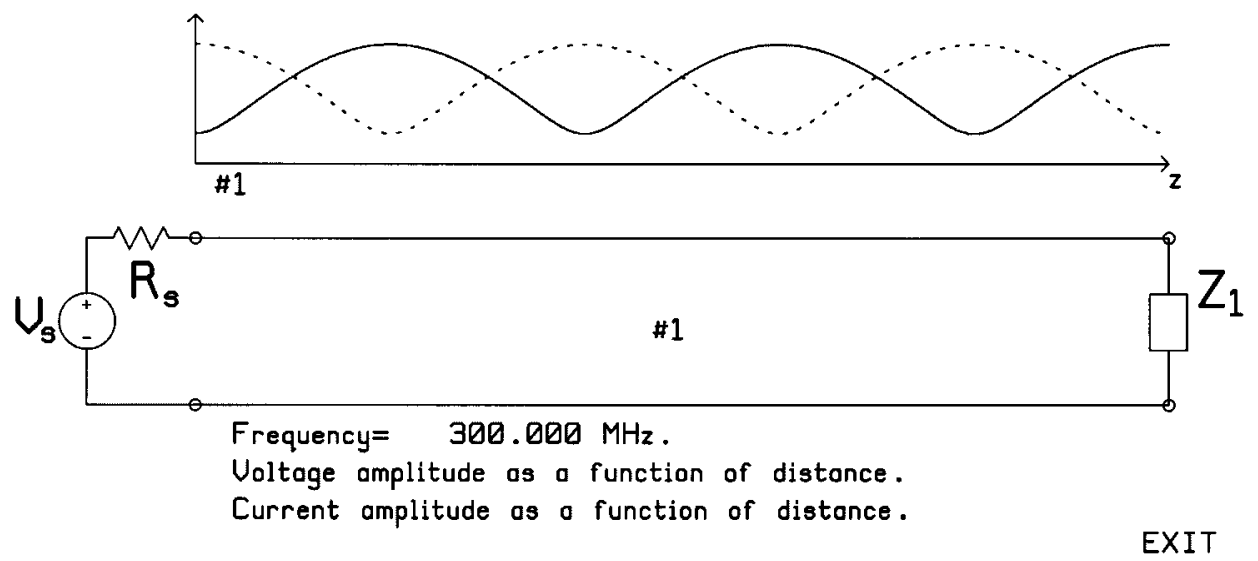

Fig. 5. Clicking on "Line \#1" in Fig. 3 graphs the amplitude of the voltage as a function of position, with markers to read back values.

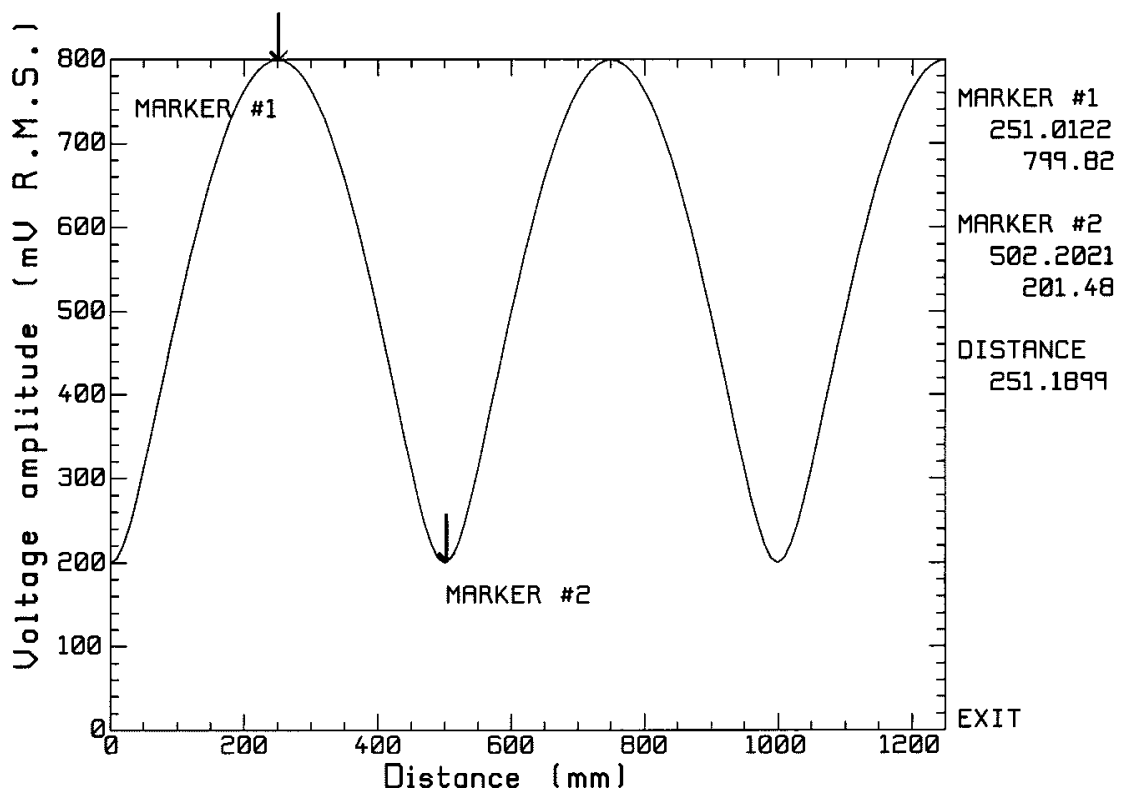

Fig. 6. The amplitude and phase of the voltage on the line for three different loads. (a) $50-\Omega$ "matched load." (b) Short-circuit load. (c) $10-\Omega$ load.

top permit the user to change line lengths and characteristic impedances, and the load values. At the bottom the user can toggle between an "admittance chart" and an "impedance chart." The center of the screen has one "button" to draw a Smith chart for each transmission line making up the circuit. There is only one transmission line in our simple circuit, so click the mouse on "Line \#1" to get the Smith chart in Fig. 8. The program offers a "sparse grid" and a "dense grid," with a button for toggling between grids at the lower right. The "dense grid" is effective on the computer screen but cluttered for hard copy, hence the "sparse grid" has been used here. To ensure that the Smith chart circle is round on the screen, the program provides an "aspect ratio" button in the lower right corner. Clicking the button obtains a menu that allows the user to adjust the roundness of the Smith chart circle on the screen, by trial and error. Values such as 1.05 or 1.1 compensate for the monitor vertical sweep adjustment on the author's computers. The program writes a file called "trline.set" to save the value of the aspect ratio for the next time that the program is run.

The Smith chart axes in Fig. 8 are labeled with "Real $\Gamma$ " and "Imag $\Gamma$ " to remind students that the chart is a complex- $\Gamma$ plane. Fig. 8 illustrates the use of the chart to transform a load impedance of $200 \Omega$ towards the generator a distance of 0.45 wavelengths by showing the circle a student would draw on a paper chart. The program reports the load reflection coefficient, the load impedance, the input reflection coefficient, and the input impedance of $82.224+j 90.619 \Omega$. To see the corresponding admittance chart, click the mouse on "switch to admittance chart" in Fig. 7, then on "Line \#1." TRLINE's easy-to-use Smith chart calculator should not replace the use of the paper Smith chart in homework exercises, as the program is not available to students in the examination! TRLINE serves to verify the student's results. 


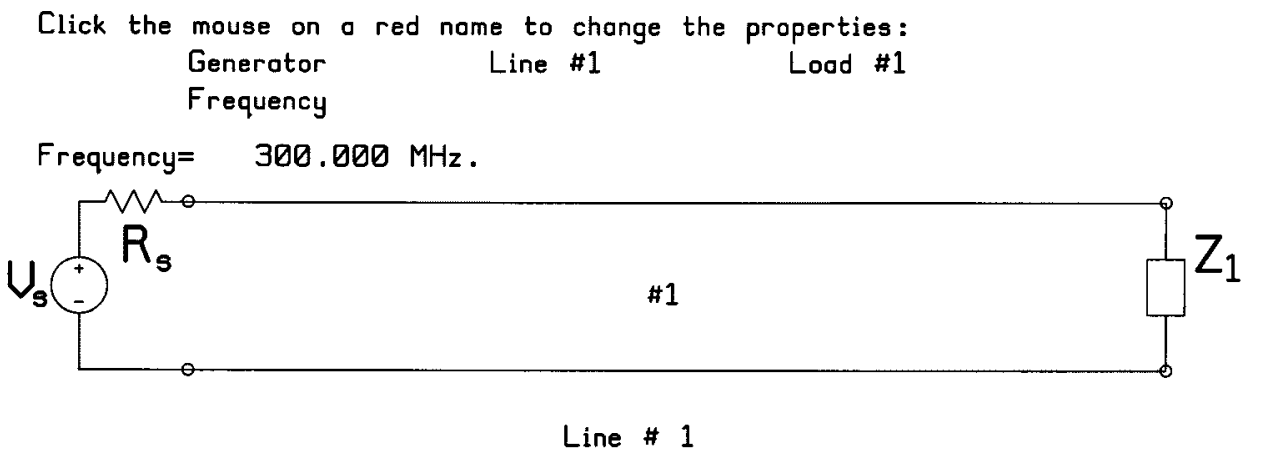

Click the mouse on a transmission line name (RED) to draw the Smith Chart.

Chort Type: Admittance

Switch to an impedance chart.
EXIT

Fig. 7. The Smith chart menu has buttons to toggle between an impedance and an admittance chart, to plot the chart for each transmission line, and to change the values of the line impedances and of the load impedances.

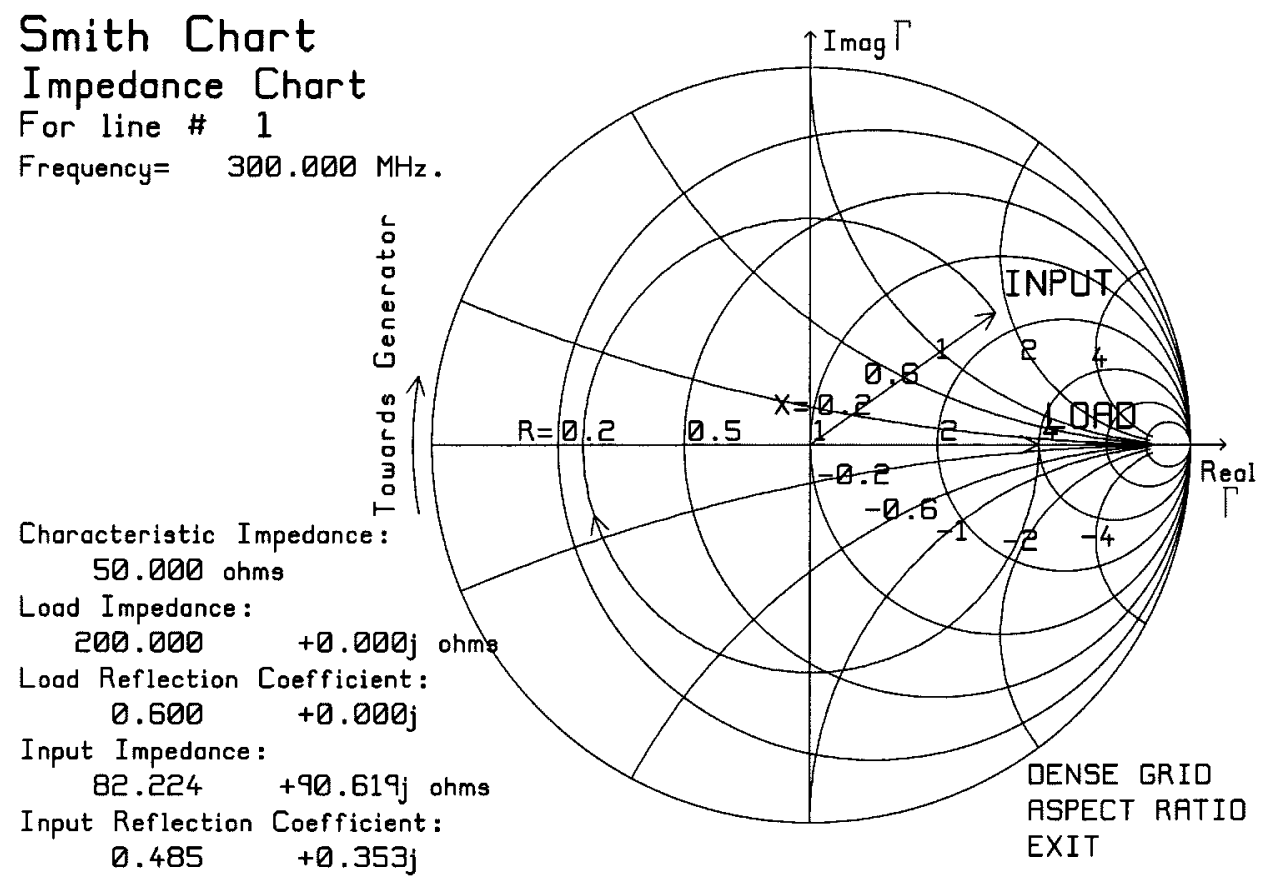

Fig. 8. The Smith chart display for the circuit of Fig. 3 in the impedance format.

\section{VOLTAGe, CURRENT, AND POWER}

Program TRLINE provides other simple circuit topologies for use with homework exercises. The branching transmission line with two loads of Fig. 9 provides a good exercise in transforming impedances back to the junction, combining in parallel, then transforming back to the generator. The student must then work forward, starting with the voltage at the input to the line, to find the voltage at the junction, and thus the power delivered to each load. The Smith chart is used to verify the student's answers for the input impedances. Clicking "find voltages, currents, and power" in the menu of Fig. 2 brings up the "voltage and power" menu of Fig. 9. Clicking the mouse on the (red) buttons for "generator," "Line \#" or "Load \#" reports the voltages, currents and powers for that part of the circuit. Fig. 10 shows the report for "Line \#1." It includes the amplitude and phase of the voltage and current at the input and output of the transmission line. The input power and output power are given, which are always equal since the lines in TRLINE are lossless. Also the input impedance and load impedance are reported.

\section{EVALUATING THE FREQUENCY BANDWIDTH}

A typical specification for a microwave component calls for a return loss better than a given figure over a given bandwidth. To illustrate the assessment of bandwidth we can design a power splitter with a quarter-wave transformer, using the circuit of Fig. 11. Lines \#1, 3, and 4 are 50- $\Omega$ lines, and the loads $Z_{1}$ and $Z_{2}$ are matched. The input impedance at the junction of lines 3 and 4 is $25 \Omega$, and so the transformer section must have a characteristic impedance $\sqrt{50 \times 25}=35.355 \Omega$. Students can verify 


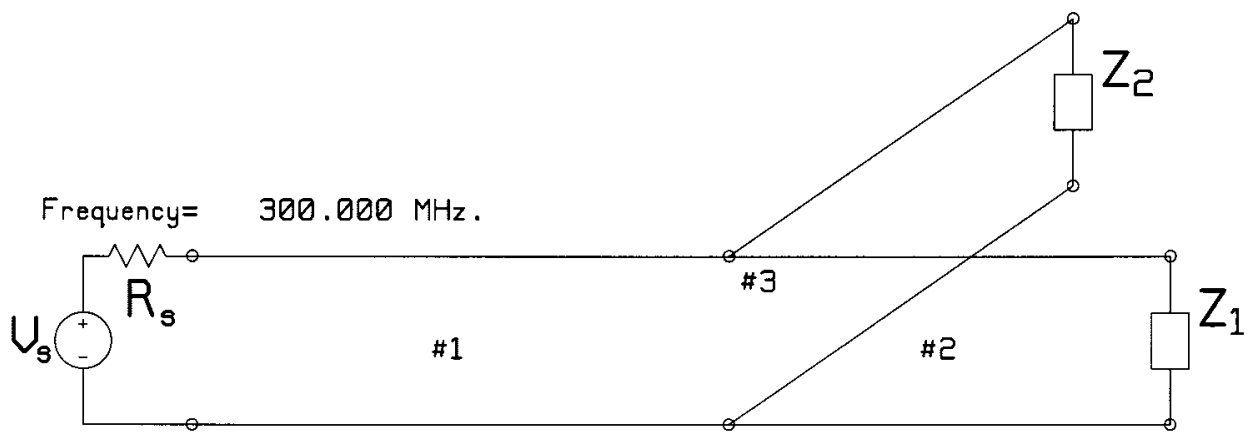

Click the mouse on o name (in red) to report the voltoge and power:

$\begin{array}{llr}\text { Generator } & \text { Line } \# 1 & \text { Lood } \# 1 \\ & \text { Line } \# 2 & \text { Lood } \# 2 \\ \text { Line } \# 3 & \\ \text { Toggle reporting odmittance or impedance. }\end{array}$

Fig. 9. The voltage and power menu lets the user find out the voltage, current, and power anywhere in the circuit.

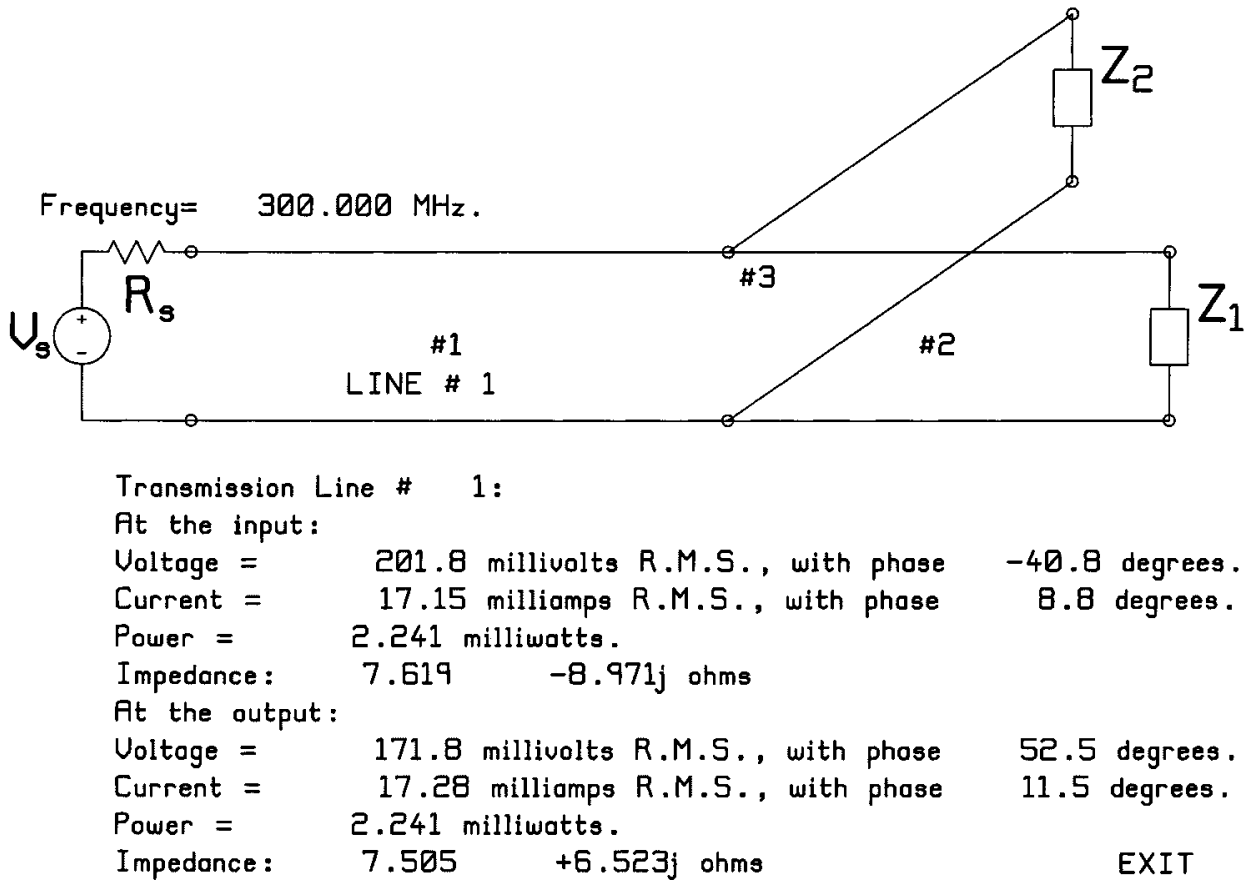

Fig. 10. Report of the voltage, current, and power for load \#1.

that this design obtains a match by drawing the voltage on each line as a function of position, as in Fig. 11, and noting that the input VSWR is 1.0000 . The smooth transition of the voltage in the transformer is of considerable interest.

A hand calculation of the bandwidth of the power splitter using a paper Smith chart is tedious and teaches little that is new once the basic Smith chart transformations are mastered. With TRLINE, bandwidths are rapidly assessed. Choosing "Plot a parameter as a function of frequency" in the menu of Fig. 2 gets the "frequency sweep" menu shown in Fig. 12. To graph the return loss at the input port, click the mouse on "return loss" after "Choose a parameter. .." then on "\#1" after "Choose a port." TRLINE uses the term "port" for the junctions in the transmission line circuit. Click on "Specify the range..." to set the starting frequency, the stopping frequency and the number of steps for computation of the return loss. Then click "Calculate..." to obtain Fig. 13 showing the return loss as a function of frequency. The graph has two "markers" which can be moved with the mouse to read back values from the graph, reminiscent of the markers available on a network analyzer. To determine the bandwidth for a return loss better than -20 $\mathrm{dB}$, click the mouse on "Marker \#1" then click again on the curve at roughly the $-20 \mathrm{~dB}$ level below the center frequency. Similarly, move marker \#2 to approximately $-20 \mathrm{~dB}$ above the center frequency. Then click on "snap menu" in the lower right corner, and type the "snap value" as -20 dB. Type "F10" and the program snaps the markers to $-20 \mathrm{~dB}$ and reports the bandwidth as approximately $110 \mathrm{MHz}$. 


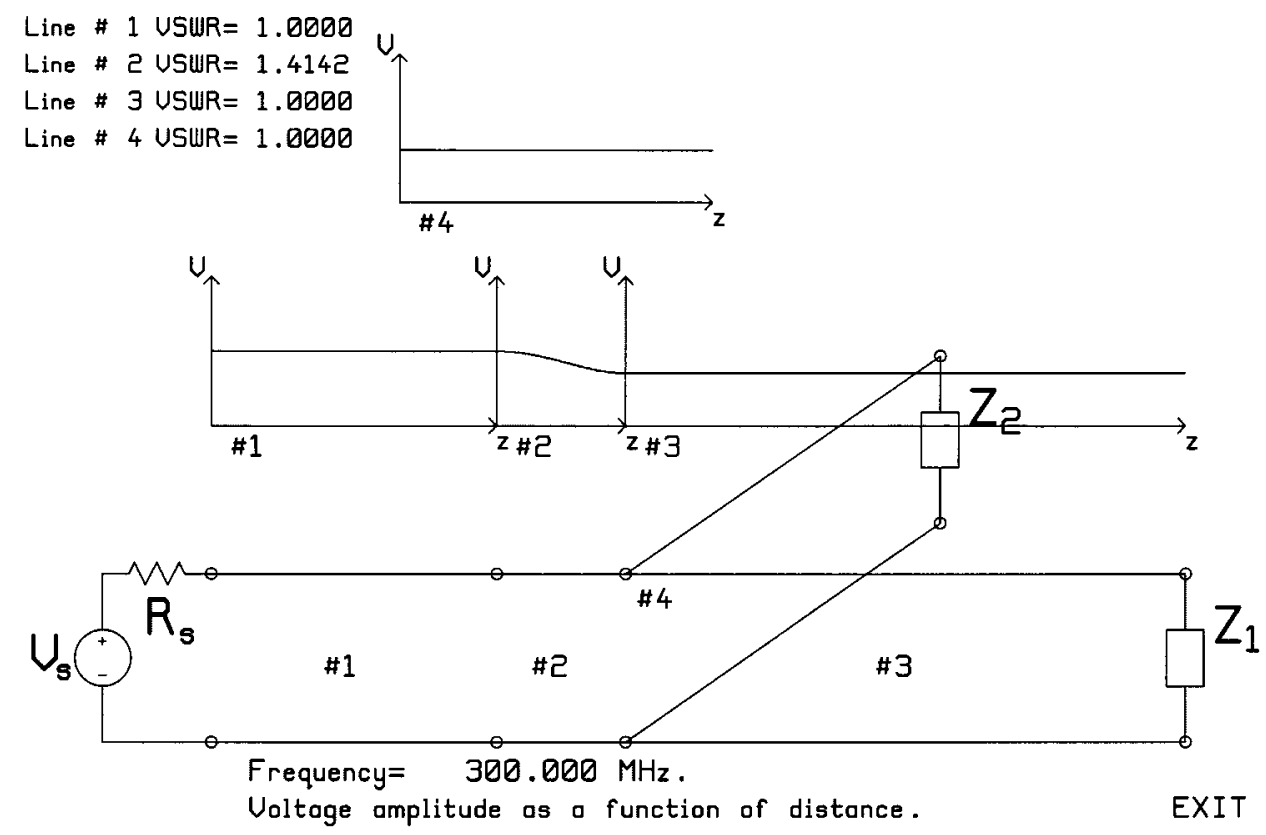

Fig. 11. The power splitter circuit template, showing the voltage on each transmission line when the impedance of the quarter-wave transformer is correctly set.
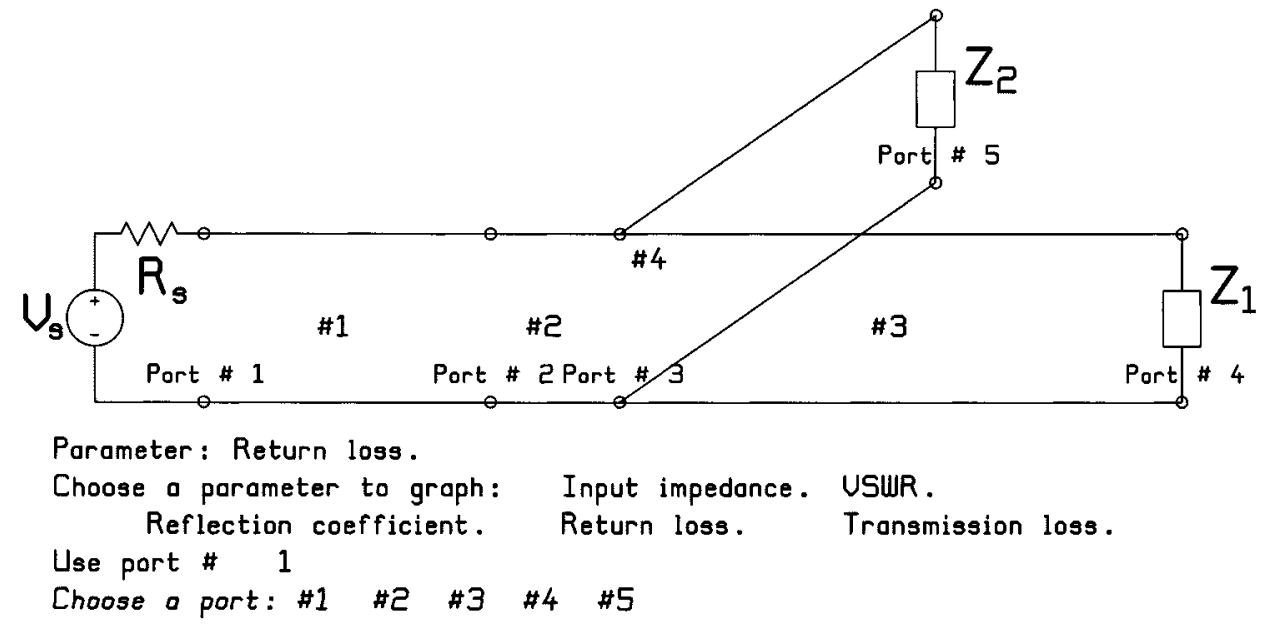

Fig. 12. The "frequency sweep" menu for graphing return loss, transmission loss, reflection coefficient, VSWR, or input impedance as a function of frequency.

\section{QUARTER-WAVE TRANSFORMERS}

The simple quarter-wave transformer used above is usually presented in the introductory "fields and waves" course, and sometimes extended to a two-step transformer [1]. In the "microwave engineering" course, matching over a wider bandwidth is investigated using three- or four-step transformer circuits [3], [4]. For a two-stage transformer matching an input line of characteristic impedance $Z_{1}$ to a line and load of impedance $Z_{4}$, choose [1]

$$
Z_{3}=\sqrt[4]{Z_{1} Z_{4}^{3}}
$$

and

$$
Z_{2}=\sqrt{Z_{1} Z_{3}}
$$

Using the frequency-sweep function in TRLINE, it can be determined that the bandwidth for a return loss of $-20 \mathrm{~dB}$ or better is about $214 \mathrm{MHz}$, much wider than the single-stage transformer's bandwidth of $110 \mathrm{MHz}$.

A three-step quarter-wave transformer can attain an even higher bandwidth. Collin [3] and Pozar [4] provide design equations for two, three, and four step Chebyshev transformers. Students can be asked to design a three-stage transformer for a return loss of $-20 \mathrm{~dB}$, or reflection coefficient $\Gamma=0.1$. The resulting bandwidth is about $371 \mathrm{MHz}$. Students can be asked to verify the relationship between the ripple level and the bandwidth of the transformer. Thus the design is repeated for a reflection coefficient $\Gamma=0.05$, or return loss $-26 \mathrm{~dB}$. The line impedances are found from [4] as $57.375,70.710$ and $87.145 \Omega$ to match a $50-\Omega$ source 


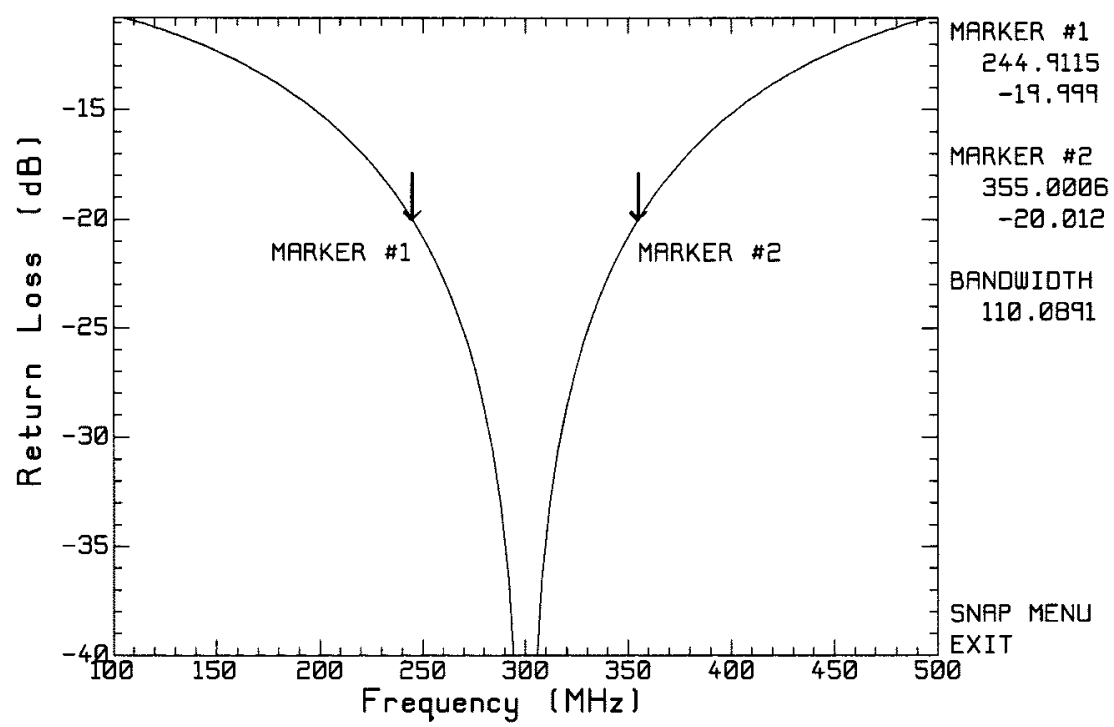

Fig. 13. The return loss of the power splitter with the markers positioned to show the bandwidth for a return loss of $-20 \mathrm{~dB}$ or better.

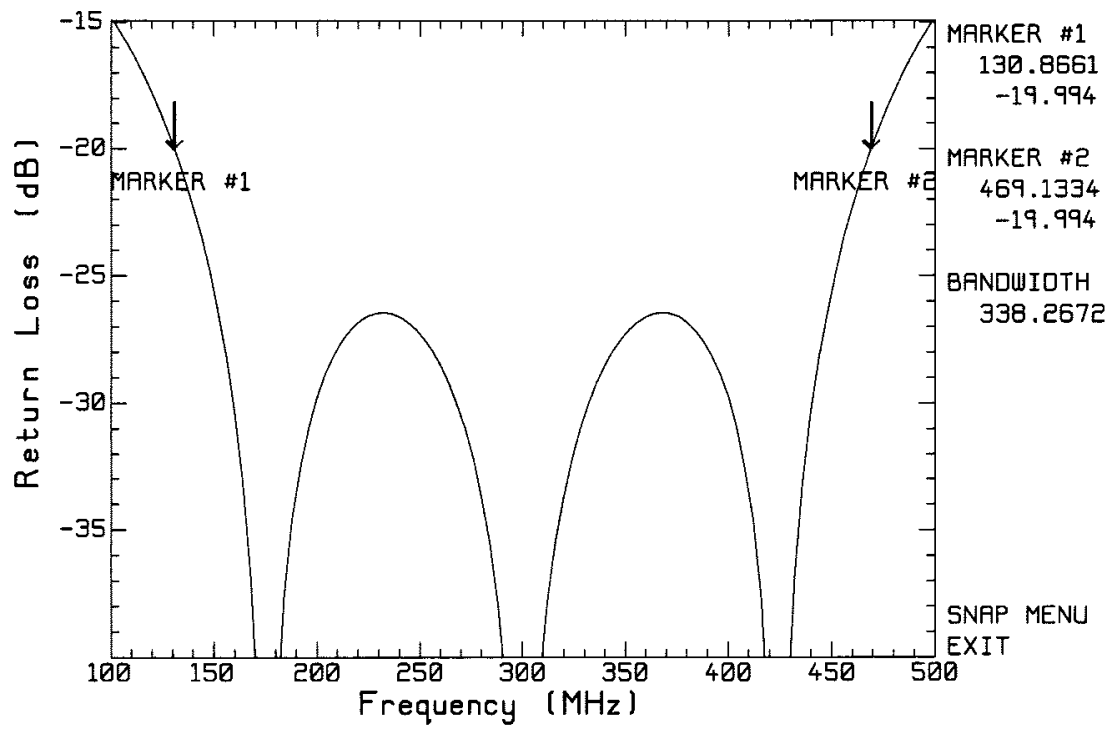

Fig. 14. The return loss of the three-stage quarter wave transformer.

to a $100-\Omega$ load. The return loss is shown in Fig. 14, and is an "equal-ripple" response as expected. The markers in Fig. 14 can be used to verify the ripple level to be -26.445 $\mathrm{dB}$. The bandwidth for a return loss better than $-20 \mathrm{~dB}$ is now $338 \mathrm{MHz}$, narrower than the design when the ripple level was permitted to rise to $-20 \mathrm{~dB}$. Students may wish to compare a two-step Chebyshev transformer with Kraus' design given above.

To try a four-step transformer, the menu of Fig. 1 provides a circuit topology consisting of six transmission lines in series with shunt loads across each junction. By setting the loads to values much larger than the characteristic impedance of the lines, the loads are "open circuit" and the four-step quarter-wave matching transformer is modeled. This circuit "template" can also be used to simulate a transmission line feeding a series of antenna elements, to compute the line lengths required to attain a given phase relationship of the antenna feed voltages.

\section{STUb Matching}

Program TRLINE provides circuit templates for single, double, and triple-stub matching. Students will design stub matching circuits using a paper Smith chart, and then test their design and evaluate the bandwidth of the match using TRLINE. The design can also be done directly with the program.

\section{A. Single Stub Matching}

Fig. 15 shows the circuit for single-stub matching. A match in line \#1 can be achieved by choosing the length of line \#2 to the load, and the length of the stub, line \#3. The wave speeds of the lines are 300 meters per $\mu \mathrm{s}$, and the characteristic impedances are $50 \Omega$. For a load of 73-j41 $\Omega$, the student uses a paper Smith chart to determine that line \#2 should be of length of $0.096 \lambda$ and the stub of length 0.145 . Graphing the voltage as a function of position shows the match is imperfect with a 


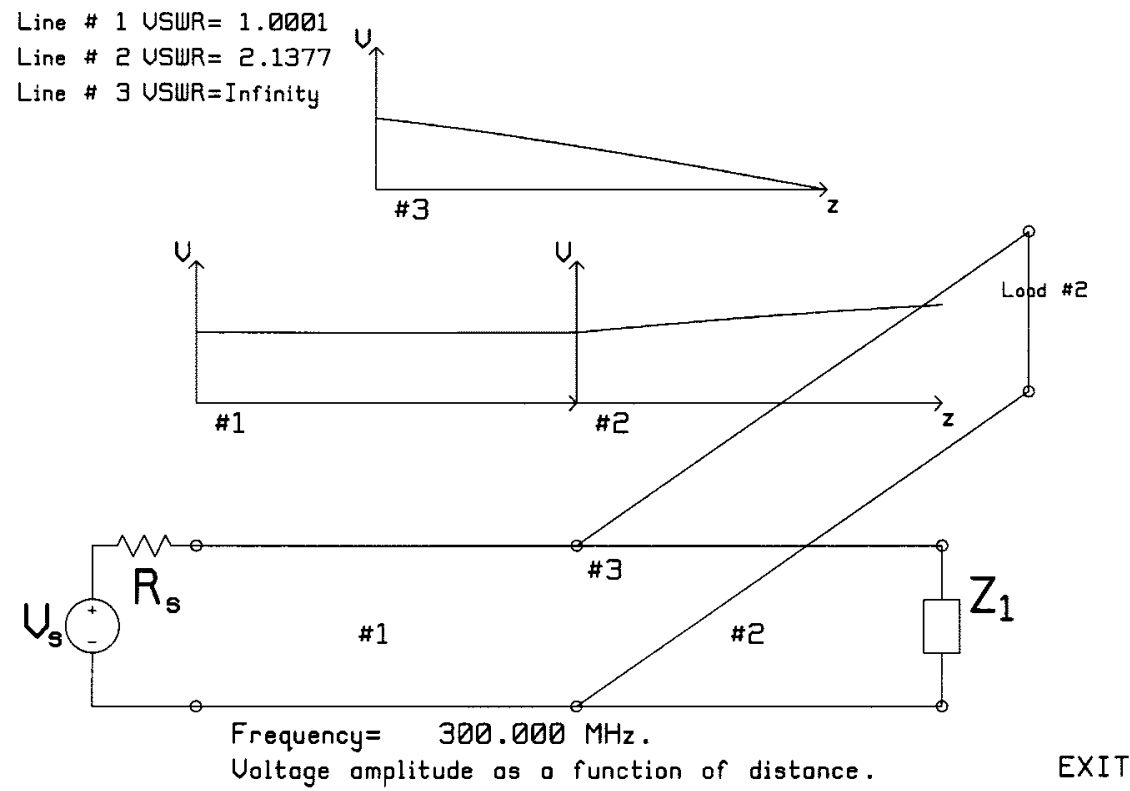

Fig. 15. The single-stub matching circuit, showing the voltage amplitude on each of the transmission lines.

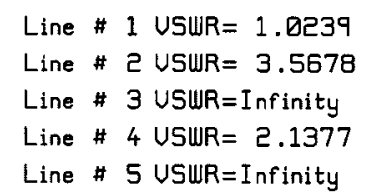

Line \# 5 USUR=Infinity
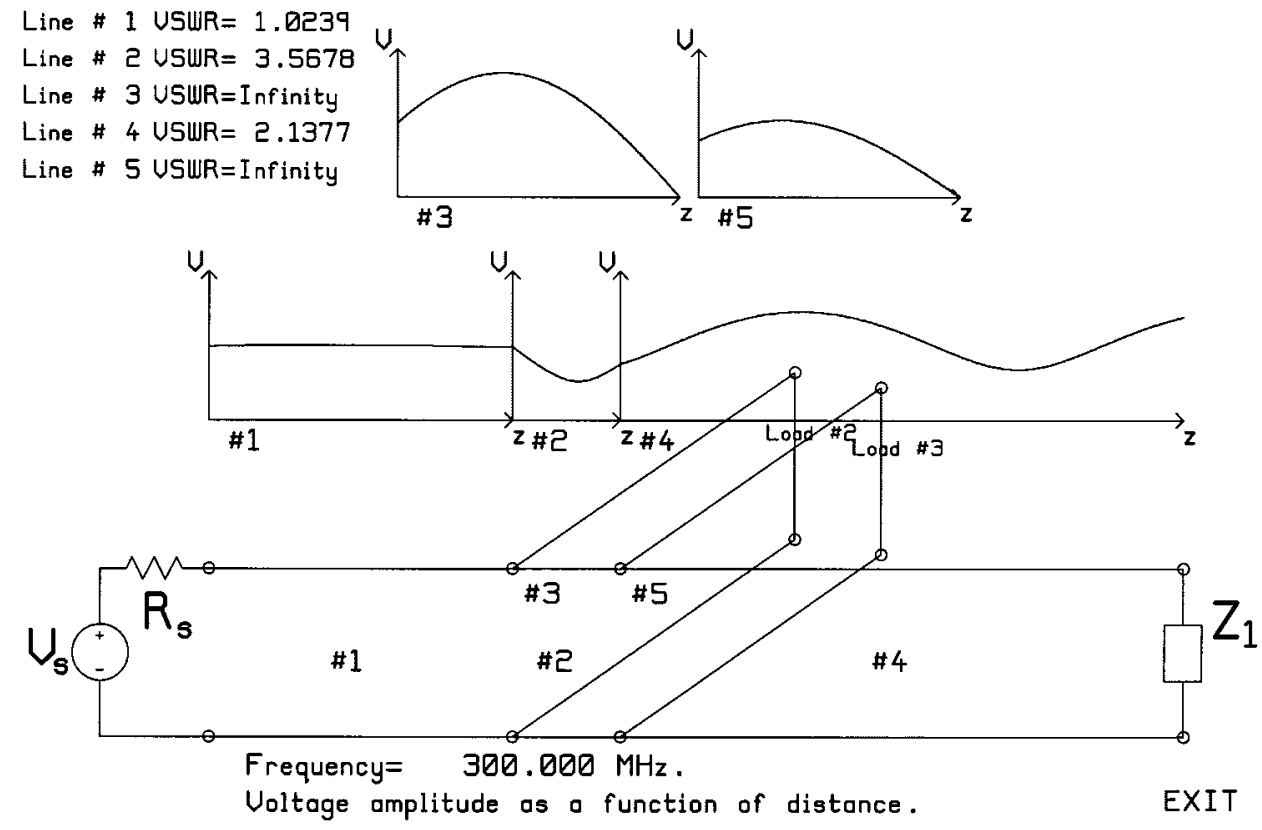

Fig. 16. Double-stub matching showing the amplitude and phase of the voltage on each of the lines.

VSWR of 1.0037. The Smith chart for line \#2 shows that the input admittance is $20.037+15.577 j \mathrm{mS}$. Because the real part differs from exactly $20 \mathrm{mS}$, the line length needs to be adjusted. The "properties button" for "Line \#2" on the Smith chart menu can be used to make small changes to the length of the line connecting the stub junction to the load, and the Smith chart used to monitor the resulting change in input admittance. It will be found that a length of $0.09582 \lambda$ gets an admittance of 20.001 $+15.563 j \mathrm{mS}$. Thus the stub, line \#3, should have an input admittance of $-15.563 j$, but with length $0.145 \lambda$ then admittance is $-15.514 j \mathrm{mS}$. Again, the Smith chart menu can be used to make small changes to the length of the stub, until the Smith chart shows that the input admittance is $-15.564 j$ for a stub length of
$0.14475 \lambda$. Then the voltage as a function of distance is graphed, as in Fig. 15, which reports the input VSWR to be 1.0001. The frequency sweep menu can be used to determine the bandwidth for a return loss of $-20 \mathrm{~dB}$ or better to be $59.6 \mathrm{MHz}$.

Students should be encouraged to explore the other solution to the single-stub-matching problem, using a line of length $0.288 \lambda$ and a stub length of $0.355 \lambda$. This design gets a VSWR of 1.0124. But the bandwidth of $22.7 \mathrm{MHz}$ is much narrower. Refining the solution slightly increases the bandwidth.

Note that TRLINE can be used to design stub matching directly, without the aid of a paper Smith chart. Thus, the length of line \#2 is adjusted until the real part of the input admittance is as close to $20 \mathrm{mS}$ as possible. Then the length of the stub, 

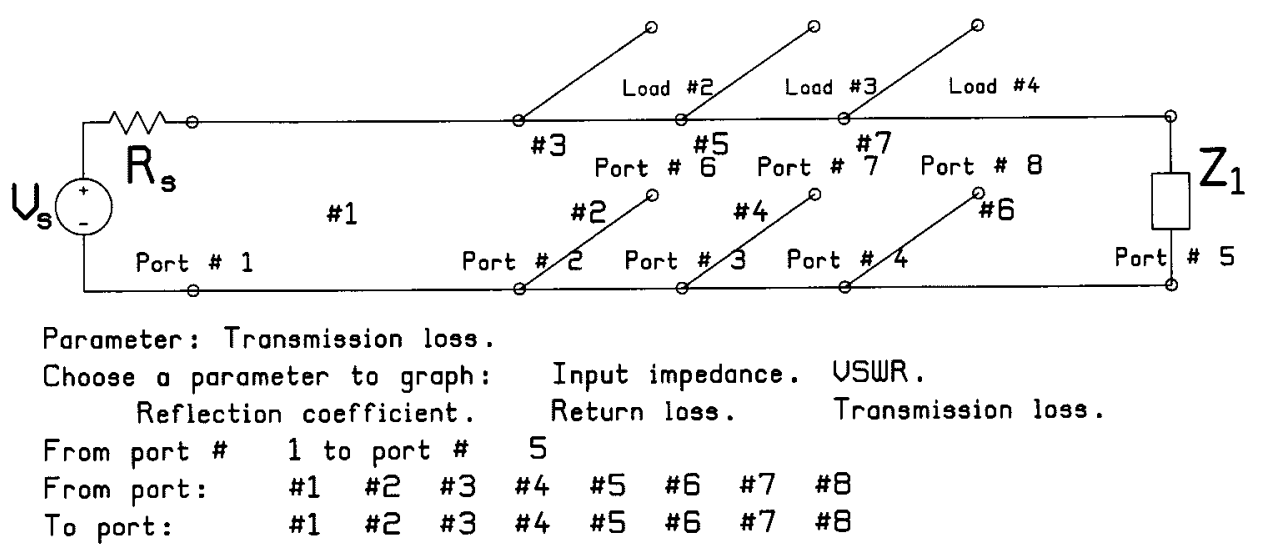

Specify the ronge of the frequency sweep.

Colculate the frequency response and graph the result. EXIT

Fig. 17. The "triple-stub matching" circuit template can model a low-pass or a bandstop filter by setting the stub terminations to open circuits.

line $\# 3$, is adjusted such that the stub susceptance cancels the susceptance at the input of line \#2. But students will not have access to TRLINE in the examination, and so must learn the use of a paper Smith Chart.

\section{B. Double Stub Matching}

Fig. 16 shows the circuit template for double stub matching. Line \#3 is the first stub, and line \#5 is the second. With the stub separation fixed at $0.125 \lambda$ and a load of $73-j 41 \Omega$ located $065 \lambda$ from the first stub, the student uses a paper Smith chart to determine that stub \#1 should be of length $0.368 \lambda$, and stub $\# 2,0.399 \lambda$. Then program TRLINE is used to see how close the input admittance of each section of transmission line is to the expected value. For line \#2 between the stubs, the input admittance is $20.386-27.447 j \mathrm{mS}$, so the stub lengths need to be refined. Using the Smith chart menu, the length of line \#5 (stub \#2) is changed in small steps until the input admittance is $20.001-27.881 j \mathrm{mS}$, for a stub length of $0.3722 \lambda$. Students can use the "interval halving" algorithm usually taught in the numerical methods course to rapidly refine the stub length. The length of line \#3(stub \#1) is adjusted to $0.40096 \lambda$ to make the input admittance $0+27.880 j$. The input VSWR is then 1.0001. The bandwidth is $9.7 \mathrm{MHz}$, much less than for single-stub matching. There is a second solution to this problem on the Smith Chart, using stubs of length $0.204 \lambda$ and $0.338 \lambda$, for a VSWR of 1.052 and a bandwidth of $16.7 \mathrm{MHz}$. Students can be asked to explore the effect of separating the stubs more widely on the bandwidth of the match.

TRLINE offers a circuit template for triple-stub matching. If the separation of the stubs is fixed, then three stub lengths must be chosen, and there is no unique solution. Students can be posed the challenge of designing a matching circuit to meet a minimum bandwidth for return loss better than, say $-20 \mathrm{~dB}$. If the stub separations can also be freely chosen, then there are five parameters. The problem is akin to designing a bandpass filter, but one that must operate into an unmatched load.
VIII. FILTERS

\section{A. Low-Pass Filter}

Students taking the elective "microwave circuits and devices" course in their final year often study microwave filter design. Pozar [4] presents filter design by the insertion-loss method and includes several examples. A low-pass filter is designed for a system of characteristic impedance $50 \Omega \mathrm{s}$. The low-pass filter uses three open-circuit stubs of length one-eighth wavelength, spaced one-eighth wavelength apart. The characteristic impedances of the stubs and of the two interconnecting lines are chosen for a 4-GHz cutoff frequency, with an equal-ripple response in the pass band of ripple level $3 \mathrm{~dB}$. To calculate the frequency response of this low-pass filter with TRLINE, use the "low-pass filter" circuit template from Fig. 1. This template is identical to the triple-stub matching circuit, but terminates the three stubs with resistive loads of $100000 \Omega$ to get "open circuits," as shown in Fig. 17. To model the low-pass filter, the input line, \#1, and the output line, \#6, are set to characteristic impedances of $50 \Omega$, and the load to $50 \Omega$. The lengths of the three stubs, lines \#3, 5, and 7, and of the lines between the stubs, \#2 and 4, are set to one-eighth wavelength at $4 \mathrm{GHz}$. The characteristic impedances of the lines between the stubs, lines \#2 and $\# 4$, are set to Pozar's values of $217.5 \Omega$, and of the stubs, lines $\# 3,5$, and 7 , to $64.9,70.3$, and $64.9 \Omega$, respectively.

To compute the filter response, click the mouse on "transmission loss" in TRLINE's frequency-sweep menu in Fig. 17. Ports to be used for the transmission loss calculation must be specified, so click \#1 following "from port" and \#5 following "to port" to ask for the transmission from the generator to the load. Then set the frequency range to 0 to $16,000 \mathrm{MHz}$, and click "calculate" to obtain the transmission loss as a function of frequency shown in Fig. 18. The markers can be used to verify that at $2.3 \mathrm{GHz}$ the transmission loss is $3 \mathrm{~dB}$, confirming the figure specified in the design. Also, the markers can be used to find that the highest frequency for a $3 \mathrm{~dB}$ transmission loss is 4 $\mathrm{GHz}$. The filter has a $20 \mathrm{~dB}$ transmission loss at $5.065 \mathrm{GHz}$. The first repetition of the filter's response due to the periodic nature of the Richard transformation is seen [4]. 


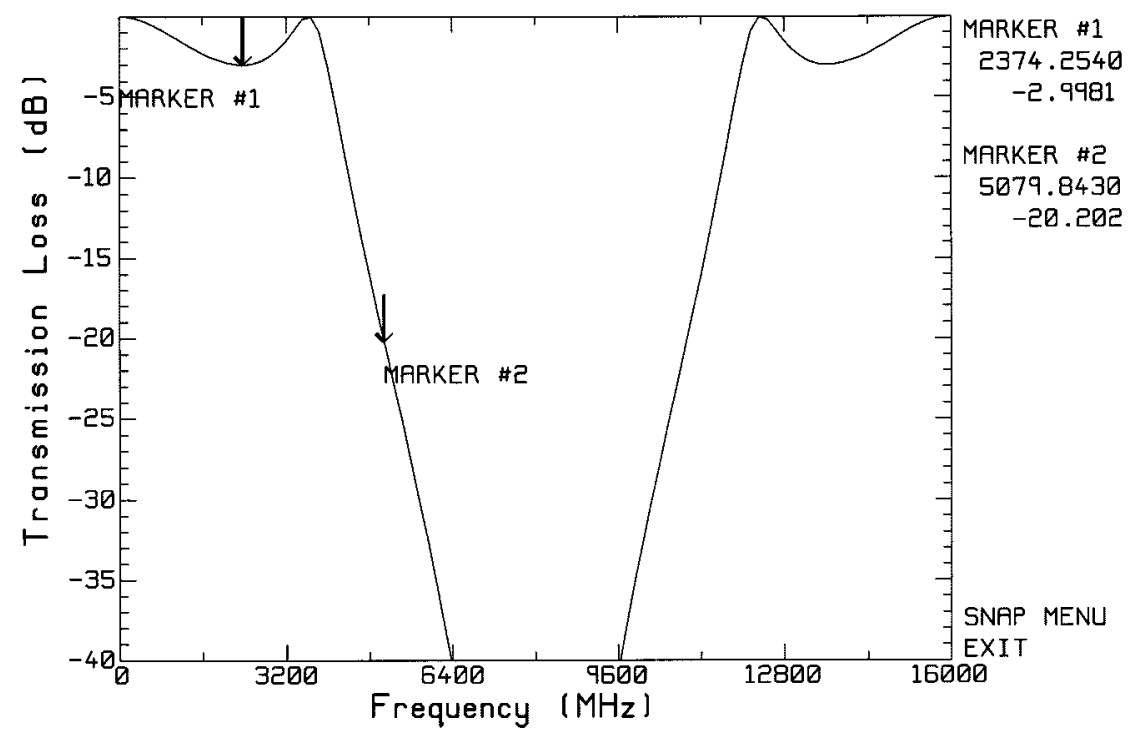

Fig. 18. The transmission loss of an equal-ripple low-pass filter.

It is instructive to graph the voltages on the transmission lines in the pass band and in the stop band, shown in Fig. 19 top and bottom, respectively. At $3638 \mathrm{MHz}$, part (a), the transmission loss is only $-0.03 \mathrm{~dB}$ and the input is quite well matched. There is a voltage-maximum at the junction of lines \#2 and \#4. Graphing the voltage as a function of distance on the individual transmission lines permits the markers to be used to find the maximum line voltages. Thus at the junction of lines \#2 and \#4 the voltage is $1.54 \mathrm{~V}$, compared to $0.5 \mathrm{~V}$ on the input line, and at the end of stub \#2 (line \#5), 2.03 volts is found, four times the voltage at the input. Fig. 19 at bottom shows the line voltages in the stop band at $5065 \mathrm{MHz}$, where the output voltage is 20 $\mathrm{dB}$ below the input voltage. A large standing wave on the input line and an output wave of amplitude $1 / 10$ the incident wave is seen. Stub \#1 has the largest voltage, but it is only $1.33 \mathrm{~V}$, lower than at $3638 \mathrm{MHz}$. Thus the largest voltages may not occur in the stop band. Also, since the voltages can be several times the input voltage, in a high-power device the voltages at the junctions and along the stubs may limit the power-handling capabilities of the filter.

\section{B. Bandstop Filter}

Pozar [4] gives the design of a bandstop filter realized using open-circuited stubs. The filter is to have a center frequency of $2.0 \mathrm{GHz}$, a bandwidth of $15 \%$, with an equal-ripple response of ripple level $0.5 \mathrm{~dB}$. Using a nominal impedance of $50 \Omega$, Pozar calculates the required impedance for quarter-wave transmission line stubs separated by a quarter-wavelength. To model the bandstop filter with TRLINE, enter the line lengths and impedances into the circuit template of Fig. 17. The lengths of lines \# 2, 3, 4, 5 and 7 are set to a quarter-wavelength at the nominal frequency of $2000 \mathrm{MHz}$. The stub impedances are set to Pozar's values of $265.9 \Omega$ for line \# 3 and \#7, and $387.0 \Omega$ for line \#5. Then compute the transmission loss to obtain the graph in Fig. 20, which is similar to Pozar's Fig. 9.49. The student can use the markers to find the ripple in the pass band to be $-1.2 \mathrm{~dB}$, somewhat larger than the nominal ripple of $0.5 \mathrm{~dB}$ wanted from the design.

Comparing Figs. 18 and 20 show that the "low-pass" and "bandstop" filters are not essentially any different. The "lowpass" filter could be used as a "bandstop" filter with a rejection better than $-20 \mathrm{~dB}$ over a bandwidth of $5.87 \mathrm{GHz}$.

Although the circuit topologies are the same as the triple-stub matching circuit, the low-pass and the bandstop filter have been included in the circuit "circuit template menu," with the line lengths, impedances and terminations appropriately set. This is very convenient for classroom demonstration of the program because the filters are easily called up. Homework exercises on filters should ask students to design their own low-pass filter to a different bandwidth and ripple level, and to verify the performance with TRLINE. The program's graphs of voltage as a function to distance can be used to determine the maximum voltage on any line at any frequency as a basis for estimating the power-handling capabilities of the device.

\section{THE TRLINE PROGRAM}

\section{A. Solving the Transmission Line Circuit}

Students will be curious about how TRLINE automatically solves circuits made of interconnections of transmission lines. Some will conclude erroneously that TRLINE has a saved solution for each of the circuit templates. In fact, TRLINE assembles a matrix equation of KVL and KCL equations at each junction in the circuit. The voltage on each transmission line is modeled with a positive-going and a negative-going travelling wave

$$
V_{k}\left(z_{k}\right)=V_{k}^{+} e^{-j \beta_{k} z_{k}}+V_{k}^{-} e^{+j \beta_{k} z_{k}}
$$

where $k$ is the transmission line number, $k=1, \cdots, N$, and $N$ is the number of transmission lines. The complex amplitudes of the voltage waves on line $\# k$ are $V_{k}^{+}$and $V_{k}^{-}$, and $\beta_{k}$ is the phase constant for line \#k. At a junction of three transmission lines, there are two KVL equations and one KCL equation. If 

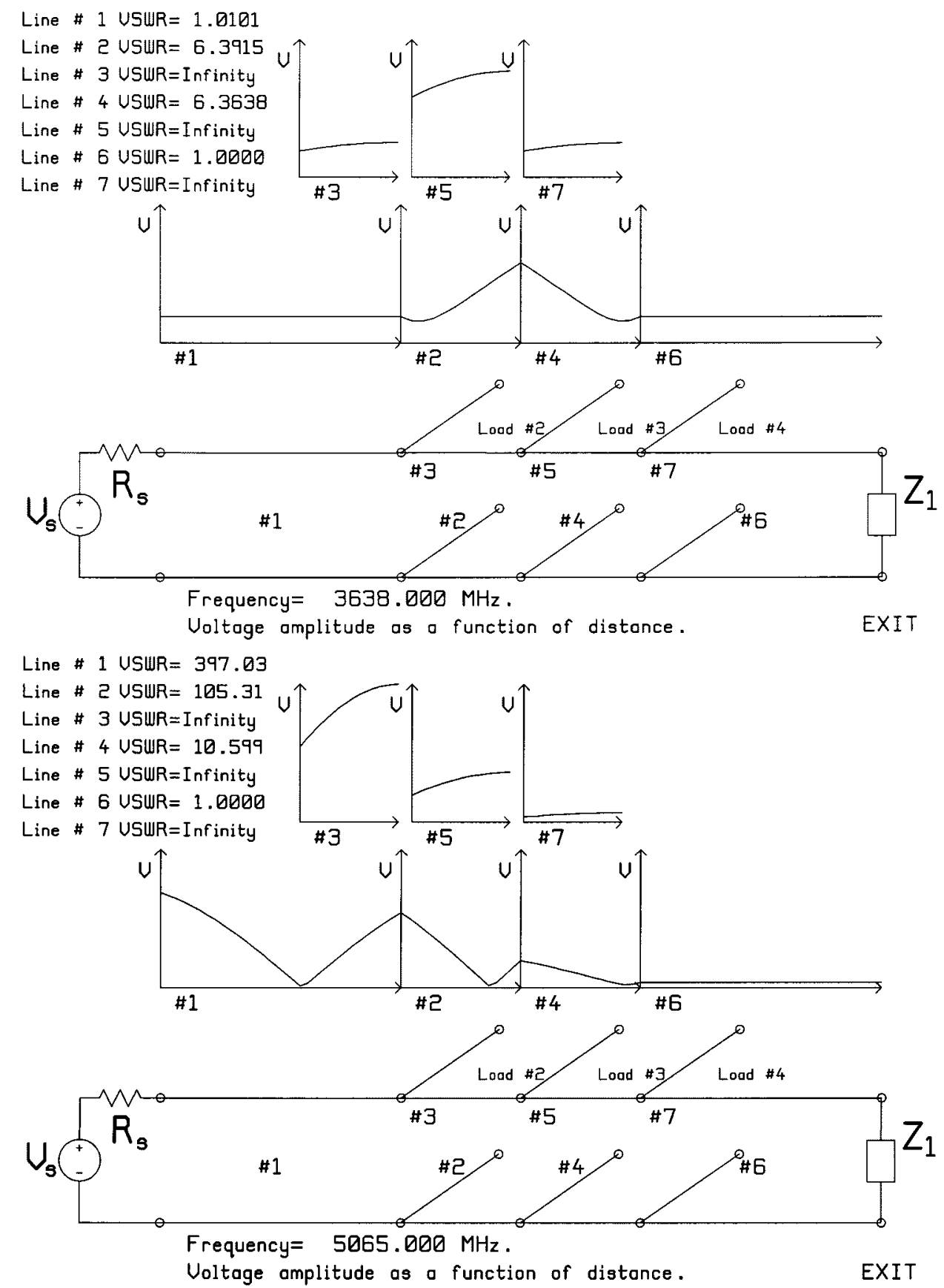

Fig. 19. The voltage on each of the lines in the pass band and in the stop band. (top) In the pass band at $3.638 \mathrm{GHz}$. (bottom) In the stop band at $5.065 \mathrm{GHz}$.

line \# $i$ enters the junction from the left, and lines \#j and \#k leave from the right, then the voltage at the end of line \#i must be the same as the voltage at the start of line \#j

$$
V_{i}^{+} e^{-j \beta_{i} L_{i}}+V_{i}^{-} e^{j \beta_{i} L_{i}}=V_{j}^{+}+V_{j}^{-}
$$

and equal to the voltage at the start of line \#k

$$
V_{i}^{+} e^{-j \beta_{i} L_{i}}+V_{i}^{-} e^{+j \beta_{i} L_{i}}=V_{k}^{+}+V_{k}^{-}
$$

where $L_{i}$ is the length of line \#i. If there is load \#m connected across the junction then the KCL equation equates the current flowing into the junction from line $\# i$ to that flowing into the load plus that flowing into lines \# $j$ and \#k

$$
\begin{aligned}
& \frac{V_{i}^{+} e^{-j \beta_{i} L_{i}}}{Z_{o i}}-\frac{V_{i}^{-} e^{+j \beta_{i} L_{i}}}{Z_{o i}} \\
& =\left(\frac{V_{i}^{+} e^{-j \beta_{i} L_{i}}}{Z_{m}}+\frac{V_{i}^{-} e^{+j \beta_{i} L_{i}}}{Z_{m}}\right) \\
& \quad+\left(\frac{V_{j}^{+}}{Z_{o j}}-\frac{V_{j}^{-}}{Z_{o j}}\right)+\left(\frac{V_{k}^{+}}{Z_{o k}}-\frac{V_{k}^{-}}{Z_{o k}}\right)
\end{aligned}
$$

where $Z_{m}$ is the load impedance, and $Z_{o i}$ is the characteristic impedance of line $\# i$. The implementation of this algorithm in TRLINE assumes that the stub branches are terminated in loads, 


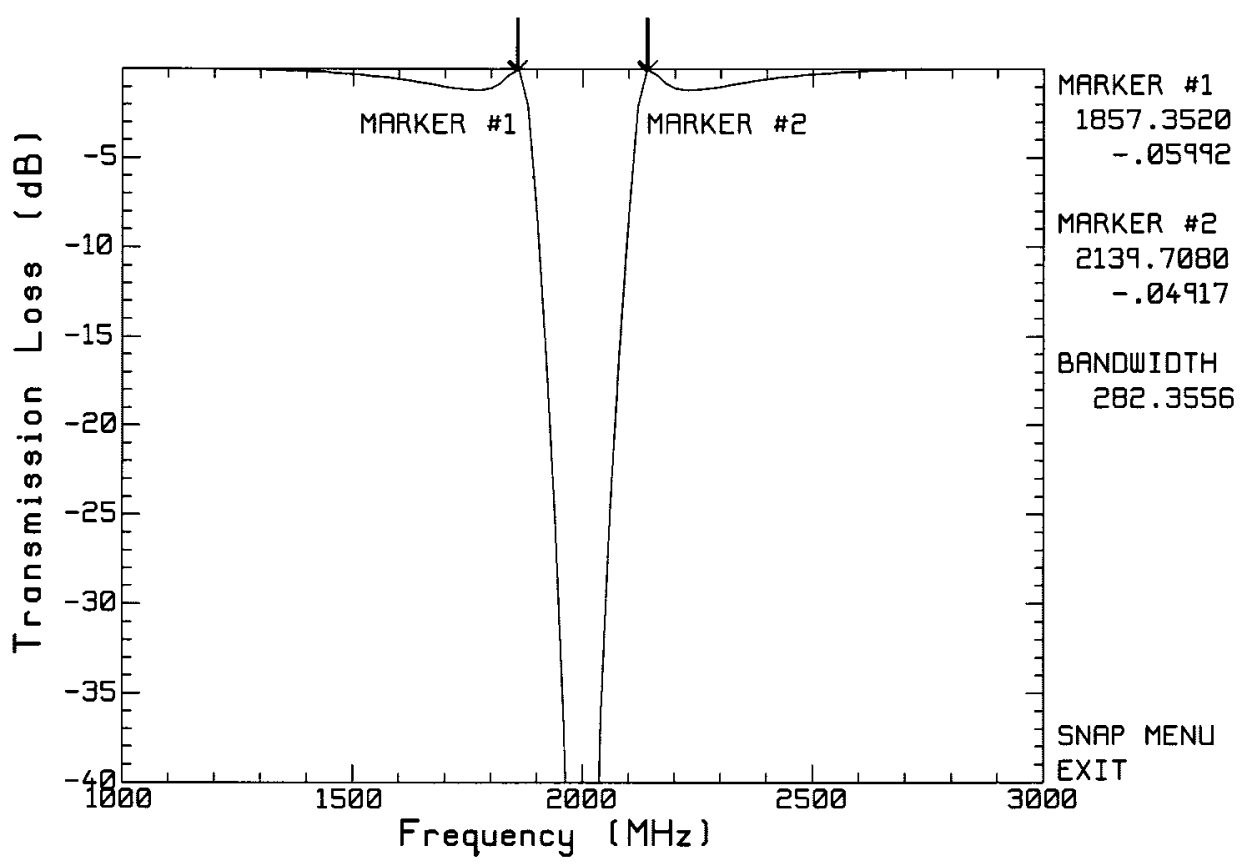

Fig. 20. Transmission loss of the bandstop filter.

so does not search for further junctions along stubs. One KVL equation is written at the generator, and one equation is written for the load terminating each stub. Gaussian elimination is used to solve the matrix equation. For 201 frequencies, the solution takes about $4 \mathrm{~s}$ on an 80486 computer at $100 \mathrm{MHz}$.

It is instructive to ask about the accuracy of the solution. Students can be asked to graph the return loss of the simple circuit of Fig. 2 with a matched load over a frequency range of say 100 to $500 \mathrm{MHz}$. It may surprise some to find that the return loss varies in a random way between -155 and $-160 \mathrm{~dB}$. The program uses single-precision arithmetic and accumulates round-off error in formulating and solving the matrix equation. A noise floor below $-150 \mathrm{~dB}$ is slightly better than might be expected with roughly seven-digit precision, since $20 \log 10^{-7}=$ $-140 \mathrm{~dB}$.

\section{B. Additional Features}

The circuit schematics drawn by TRLINE use line lengths that are proportional to the true values. As the lengths are changed the circuit diagram grows or shrinks, giving a sense of the relative size of the parts of the circuit. Sometimes portions of the circuit schematic have been permitted to overlap the graphs of line voltage, as in Figs. 11 or 15. This does not detract from the usefulness of the graphs.

After all the line lengths and characteristic impedances of a complex circuit have been entered, the circuit parameters can be saved to a file by clicking "save the circuit" in Fig. 2. The program asks for a file name and writes the file to disk with the TRLINE file extension "trl." The circuit can be recalled using "Read a saved circuit" in the menu of Fig. 1. The "trl" file is a text file that includes comments explaining the structure of the file. The enterprising student can construct new circuits not included in the "templates" menu by editing the "trl" file. Thus a circuit with four or five stubs could be created.
The upper left corner of TRLINE's window on the screen includes "edit," "print" and "options" buttons, which have not been shown in the figures in this paper. Any display that the program creates can be printed by clicking the mouse on the "print" button. Also, any display can be copied to the Windows clipboard by clicking "edit" then "copy," then pasted into a word-processor document. The "options" button lets the user change the display from color to monochrome, to visualize its appearance in a printed document. Also the background can be changed from white to black. Many of TRLINE's screens are more effective against a black background.

Whenever a frequency sweep graph is drawn, TRLINE produces a data file containing the graph in file TRLINE.RPL. This file can be used with the rectangular plotting program "RPLOT" [5] to create well-formatted graphs of impedance, reflection coefficient, return loss, or transmission loss as a function of frequency. Whereas TRLINE does not give the user any control over the format of the axes in the rectangular graph, RPLOT permits full control over axis format, labeling, titles, and so forth.

\section{Limitations}

TRLINE is not intended as a solver of transmission line circuits of arbitrary layout or complexity. Indeed, the emphasis has been put on ease of use and not on generality. Better but much more complex tools such as SPICE [6] or TOUCHSTONE [7] are available. This section briefly mentions limitations and some possible extensions of the program's functions.

TRLINE's lossless transmission lines could easily be made lossy by including the attenuation factor in the matrix equation, and adding the attenuation constant to each line's properties. To simplify the assembly of the matrix and the creation of the graphics displays, the present version of TRLINE forces stub branches to terminate in a load. It might be useful to extend the program to allow branches with a series connection of two or 
three lines terminating in a load. The present TRLINE uses constant-impedance loads. This is readily extended to parallel RLC loads whose impedance varies with frequency, useful for modeling bandpass filters. Series RLC loads could also be included if there is a need. It would be possible to include the computation of the characteristic impedance of each line at each frequency, to model waveguide components using the dominant mode of propagation only. A bigger extension of the program's capabilities would be a user interface to construct transmission line circuits. This goes somewhat beyond the objectives of the TRLINE program, as it is not intended to solve transmission line circuits of arbitrary complexity.

\section{CONClusion}

Program TRLINE is intended as an aid to teaching about transmission lines at the introductory and intermediate level. TRLINE's built-in circuit templates for many common problems and simple mouse-driven user interface let students do useful work almost at once. TRLINE provides a "laboratory" for students to test their solutions to homework problems. Program TRLINE helps establish the relationship in a student's mind between terminal properties, such as input impedance computed via the Smith chart, and the underlying voltages and current magnitude and phase as a function of position.

TRLINE introduces the possibility of refining paper stubmatching designs, quite similar to the practice in the microwave components industry of developing prototypes "on the bench" by making small dimensional changes. The bandwidth of a component is often a key specification. TRLINE provides a quick computation of return loss or transmission loss as a function of frequency, allowing homework problems to include a bandwidth specification, which gives the flavor of real component design. Filter designs can be "tuned" with TRLINE to increase the rejection or widen or narrow the bandwidth. TRLINE makes accessible the line voltages as a function of position at many frequencies, and encourages the student to relate them to the return loss or transmission loss over a frequency range.
TRLINE leads the student from simple circuits to the design of transformers and simple filters. As students progress beyond the intermediate level, a transition should be made to software which is oriented toward the design of real components. Thus PUFF [8] provides a realistic design environment for stripline components. Using TOUCHSTONE [7] at this level introduces students to a software tool often found in the workplace.

\section{REFERENCES}

[1] J. D. Kraus, Electromagnetics, 4th ed. New York: McGraw-Hill, 1992.

[2] C. R. Paul and S. A. Nasar, Introduction to Electromagnetic Fields, 2nd ed. New York: McGraw Hill, 1987.

[3] R. E. Collin, Foundations for Microwave Engineering. New York: McGraw-Hill, 1966.

[4] D. M. Pozar, Microwave Engineering. Reading, MA: Addison-Wesley, 1990.

[5] C. W. Trueman. TRLINE User's Guide. [Online]http://www.ece.concordia.ca/ trueman/trline

[6] P. W. Tuinenga, SPICE: A Guide to Circuit Simulation and Analysis Using SPICE. Englewood Cliffs, NJ: Prentice-Hall, 1988.

[7] Guild and the technical support staff, Touchstone and Libra reference. Westlake Village, CA: 3.5, EE SofInc., Dec. 1991.

[8] R. Compton and D. Rutledge, PUFF-Computer Aided Design for Microwave Integrated Circuits: Richard Compton, Cornell University, and David Rutledge, publishers. California Univ. Technol., Pasadena, Jan. 1989.

Christopher W. Trueman (S'75-M'75-SM'96) received the B.Eng. degree in 1972 and the M.Eng. degree in 1975, both from McGill University, Montreal, Canada. He received the Ph.D. degree from McGill University in 1979 with a thesis on wire-grid modeling of aircraft antennas.

He became a Lecturer at Concordia University, Montreal, Canada, in 1974. He was promoted to Associate Professor in 1985, and is currently the Associate Chair and Co-op Program Director for the Department of Electrical and Computer Engineering at Concordia. His research includes computational electromagnetics, using moment methods, the finite difference time domain method, and geometrical optics, and geometrical diffraction. He has done contract research on the scattering of standard broadcast signals from power lines, on the radar cross section of aircraft and ships at $\mathrm{HF}$ frequencies, on helix antenna analysis and design, and on the near and far fields of cellular telephones operating near the human head. He is active in the areas of code validation and computer graphics for visualization of results in electromagnetic problems.

Dr. Trueman is a member of Order of Engineers of Quebec. 